Planetary Systems in the Universe - Observation, Formation and Evolution

Proceedings IAU Symposium No. 202, (C)2004 IAU

Alan Penny, Pawel Artymowicz, Anne-Marie Lagrange, 8 Sara Russell, eds.

\title{
Chemical Composition of Planet-harboring Stars
}

\author{
G. Zhao, Y.Q. Chen, H.M. Qiu, S.K. Tang, Z.W. Li \\ National Astronomical Observatories, Chinese Academy of Sciences, \\ Beijing 100012, China \\ Department of Astronomy, Beijing Normal University, Beijing 100875, \\ China
}

\begin{abstract}
Based on the high-resolution, high signal-to-noise ratio spectra collected with the coude echelle spectrograph attached to the $2.16 \mathrm{~m}$ telescope at Beijing Astronomical Observatory, we determined the chemical abundance patterns for a sample of six planet-harboring stars. The result is used to investigate the connection between giant planet and high metallicity and to probe the influence of this process on other elements.
\end{abstract}

\section{Introduction}

The discovery of the planet surrounding a main sequence star provides us an important clue to understand the theories of the planetary system and stars. The dependence of the planet formation on stellar metallicity is of great interest based on the facts that many such stars are really metal-rich. However, there exists a few stars which was found to hold planets but has quite low metallicity. This work presents the accurate metallicities for six planet-harboring stars. The main purposes are to investigate if all our sample stars follow the planet-high metallicity connection and to know how this process acts on different elements by studying the ratios between elements with the same nucleosynthesis history.

\section{Observations and Data Reductions}

The observations were carried out with the coudé echelle spectrograph attached to the $2.16 \mathrm{~m}$ telescope at the National Astronomical Observatories (Xinglong, China). The detector was a Tek CCD $\left(1024 \times 1024\right.$ pixels with $24 \times 24 \mu m^{2}$ each in size). The spectra have a resolution around 40000 , wavelength coverage from $5600 \AA$ to $8300 \AA$, and $\mathrm{S} / \mathrm{N}$ around 200 except for $\rho$ Cnc which spectra has a lower quality.

The data reduction follows standard routines for order definition, background subtraction, wavelength calibration, radial velocity shift and continuum normalization. The equivalent widths were measured by a direct integration and Gaussian fitting depending on the line intensity. 
Table 1. Model parameters and element abundances

\begin{tabular}{lrrrrrr}
\hline Star & 47 UMa & $51 \mathrm{Peg}$ & $55 \mathrm{Cnc}$ & $70 \mathrm{Vir}$ & HD 29587 & HD 98230 \\
\hline$T_{\text {eff }}(\mathrm{K})$ & 5756 & 5649 & 5046 & 5430 & 5550 & 5796 \\
$\log \mathrm{g}$ & 4.23 & 4.27 & 4.28 & 3.92 & 4.07 & 4.43 \\
{$[\mathrm{Fe} / \mathrm{H}]$} & -0.08 & 0.26 & 0.19 & -0.02 & 0.09 & -0.35 \\
$\xi_{\mathrm{t}}\left(\mathrm{kms} \mathrm{m}^{-1}\right)$ & 1.05 & 0.45 & 1.45 & 0.75 & 0.90 & 0.85 \\
\hline$[\mathrm{Na} / \mathrm{Fe}]$ & -0.01 & -0.13 & - & -0.09 & -0.01 & -0.04 \\
{$[\mathrm{Mg} / \mathrm{Fe}]$} & -0.02 & -0.15 & -0.02 & -0.08 & -0.15 & 0.11 \\
{$[\mathrm{Al} / \mathrm{Fe}]$} & 0.19 & - & - & 0.07 & - & -0.02 \\
{$[\mathrm{Si} / \mathrm{Fe}]$} & 0.05 & 0.05 & 0.09 & 0.07 & 0.17 & 0.00 \\
{$[\mathrm{Ca} / \mathrm{Fe}]$} & 0.11 & 0.01 & -0.24 & 0.09 & 0.06 & 0.11 \\
{$[\mathrm{Sc} / \mathrm{Fe}]$} & 0.17 & 0.19 & 0.17 & 0.13 & 0.13 & -0.04 \\
{$[\mathrm{Ti} / \mathrm{Fe}]$} & -0.05 & -0.03 & -0.32 & -0.03 & -0.05 & 0.01 \\
{$[\mathrm{~V} / \mathrm{Fe}]$} & 0.01 & -0.05 & -0.02 & 0.01 & -0.07 & -0.05 \\
{$[\mathrm{Cr} / \mathrm{Fe}]$} & -0.09 & 0.14 & -0.16 & 0.03 & 0.10 & -0.00 \\
{$[\mathrm{Mn} / \mathrm{Fe}]$} & -0.06 & 0.06 & -0.11 & -0.12 & 0.09 & -0.28 \\
{$[\mathrm{Ni} / \mathrm{Fe}]$} & 0.03 & 0.20 & 0.04 & 0.02 & 0.15 & -0.02 \\
{$[\mathrm{Y} / \mathrm{Fe}]$} & - & -0.25 & -0.35 & -0.09 & -0.37 & \\
{$[\mathrm{Ba} / \mathrm{Fe}]$} & 0.01 & 0.11 & - & 0.18 & 0.02 & 0.31 \\
\hline
\end{tabular}

\section{Line Analysis}

The effective temperatures were derived from $(b-y)$ color indices using the calibration of Alonso et al. (1996). The surface gravities were accurately calculated from Hipparcos parallaxes except for HD 98230, which is estimated from photometric $c_{1}$ index because Hipparcos parallax is not available. The microturbulence velocity was determined from the abundance analysis by requiring a zero slope of $[\mathrm{Fe} / \mathrm{H}]$ vs. EW.

The abundance analysis is based on a net of flux constant, homogeneous, LTE model atmospheres taken from Kurucz (1993). The abundances were derived by requiring that the calculated equivalent widths should match the observed ones. Solar abundances calculated from the moon spectrum, were used to derive stellar abundances relative to solar values. Such differential abundances are generally more reliable than absolute abundances because many systematic errors nearly cancel out.

\section{Result and Discussions}

Fig. 1 shows the metallicities of our sample stars and the trends of individual elemental abundances with metallicities. As far as the planet-metallicity connection, we have following results: Firstly, we confirm the high metallicity for $51 \mathrm{Peg}$ and $\rho \mathrm{Cnc}$ with $[\mathrm{Fe} / \mathrm{H}]$ as high as 0.2 dex. Secondly, $70 \mathrm{Vir}, 47 \mathrm{UMa}$ and HD 29587, with metallicities from $[\mathrm{Fe} / \mathrm{H}]=-0.08$ to +0.09 are quite similar to the sun. These metallicities are still higher than most F and G-type main 

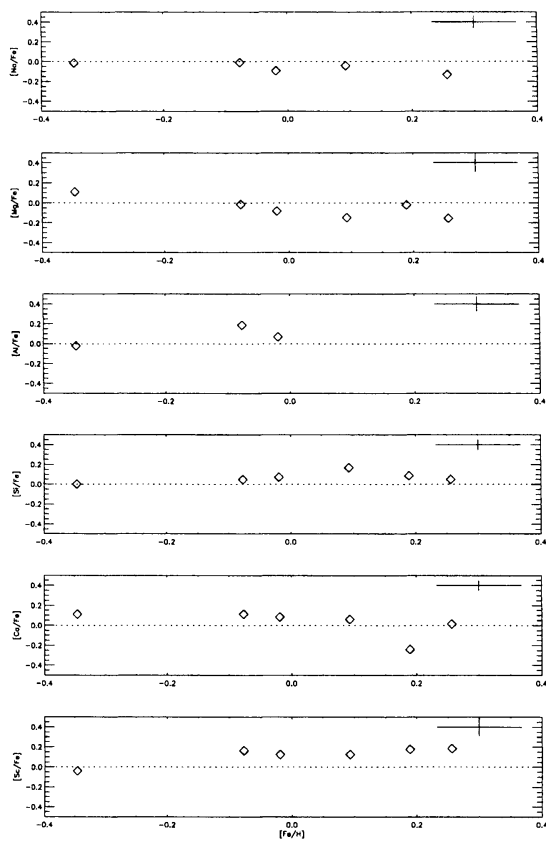
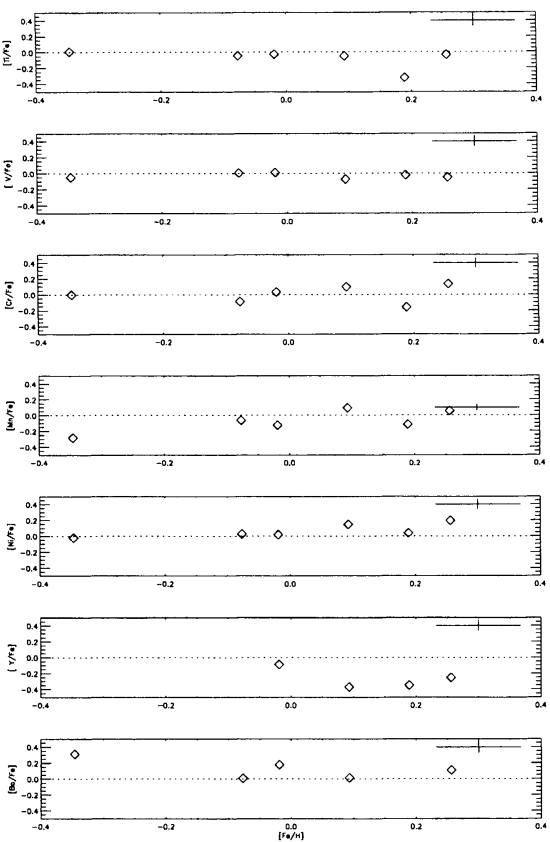

Figure 1. The trend of $[\mathrm{X} / \mathrm{Fe}]$ with $[\mathrm{Fe} / \mathrm{H}]$ for $\mathrm{Na}, \mathrm{Mg}, \mathrm{Al}, \mathrm{Si}, \mathrm{Ca}, \mathrm{Sc}, \mathrm{Ti}$, $\mathrm{V}, \mathrm{Cr}, \mathrm{Mn}, \mathrm{Ni}, \mathrm{Y}$ and $\mathrm{Ba}$. The error bar is shown in the upper-right corner of each sub-figure.

sequence stars in the solar neighbourhood with a metallicity peak at $[\mathrm{Fe} / \mathrm{H}]=$ -0.2 dex. All the above 5 stars seems to support the connection between the planet formation and stellar high metallicity. However, HD 98230 with $[\mathrm{Fe} / \mathrm{H}]=$ -0.35 is contrary to this suggestion. Together with HD 114762 , it seems that we need other explanation to understand their presence of giant planets. We found most elements are quite similar to the sun with $[\mathrm{X} / \mathrm{Fe}]$ nearly close to zero, and there is no significant trend with metallicity. It seems that the high metallicity of planet host stars is primordial since there is no special signature in other elements based on this small sample of stars. Investigation on a large sample of planet host stars is necessary. In this respect, we have started a project on abundance determination of 70 planet host stars in order to detect abundance anomalies of elements other than iron statistically.

This research was supported by the NSFC and the NKBRSF G1999075406.

\section{References}

Alonso, A., Arribas S., \& Martínez-Roger, C. 1996, A\&A, 313, 873

Kurucz, R.L. 1993, CD-ROM 13, Smithsonian Astrophys. Obs. 